\title{
Smartcard based Android Application for Public Transport Ticketing System
}

\author{
Neelam Gulrajani \\ Student, Design for Digital Experience \\ National Institute of Design \\ Bengaluru, India

\begin{abstract}
The large amount of growth in India's population has increased the demand on the transport systems. In the areas of public transport, it has become essential to consider effective technologies that will ease the system of transport. This paper presents the design of a public transport ticketing system that harnesses the functionality of the existing electronic smart cards into an Android application. This application aims to relate the Man Machine Interaction (MMI) principles of functionality, usability, simplicity, visibility, affordance and consistency for enhancing the experience of ticketing for the public transport commuters in India.
\end{abstract}

A HCl Approach

\author{
Dhananjay R. Kalbande, PhD. \\ Asso. Prof.\& HOD, Dept. of Computer Engineering \\ Sardar Patel Institute of Technology \\ University of Mumbai, India.
}

\author{
Srinath Warrier \\ Software Engineer \\ SAP Labs India Pvt Ltd. \\ Bengaluru, India
}

\section{Keywords}

Smart cards; Human computer interaction; Public transportation; Rail transportation; Mobile computing.

\section{INTRODUCTION}

Human-Computer Interaction (HCI) is the design and implementation of interactive computing systems that users can interact with. Also referred to as MMI (Man Machine Interaction), $\mathrm{HCI}$ allows us to gain a fresh perspective of joint interaction between man and machine. The most important concepts in HCI are functionality and usability. A system is said to be successful if there is a balance between both functionality and usability [1]. The research findings aim to develop such a balanced application of HCI in the arena of public transport ticketing.

The application is based on Android and thus it incorporates the simplicity and usability that Android platform has to offer. Also in the third quarter of 2011, Android OS accounted $52.5 \%$ of worldwide smartphone sales to end users (compared to $25.3 \%$ a year earlier) whereas Symbian accounted $16.9 \%$ (36.3\% a year earlier), iOS accounted $15.0 \%$ (16.6 a year earlier) and Research In Motion accounted $11.0 \%$ (15.4\% a year earlier) according to Gartner [2]. Thus implementing the application on the Android platform also ensures maximum customer reach.
The use of the smartcards ensures the amalgamation of existing functionalities of the Automatic Ticket Vending Machine (ATVM) ticketing. Presently the commuters of metropolitan cities either wait in the long waiting queues to avail the railway tickets, punch the railway coupons or utilize the ATVM machines by means of electronic smartcards. The proposed system provides an efficient, easy-to-use and a convenient alternative to the current means.

\section{USER CENTERED DESIGN OF THE APPLICATION}

Interaction design, as defined by Winograd in 1997, is the design of spaces for human communication and interaction [3]. The goal of interaction design is to develop usable products. In simple words usable implies easy to learn, effective to use and provides pleasant user experience. The application intended the interaction design to be user centered. The application projects some principles of a user centered design as follows:

2.1.1.1 Vision: The initial concept arose from the need of a user- friendly and a convenient medium of ticketing that is more intuitive than instructive.

2.1.1.2 Analyze: It was analyzed that there was a need of an on-the-go medium. Mobile phones and tablets serve this purpose. A user friendly operating system that has a maximum reach along with an application user interface that is designed for usability will suffice the user centered requirements. The use case scenario constructed during the analysis phase is as shown in Table 1:

Table 1. Use case buying a ticket

\begin{tabular}{|l|l|}
\hline Name & Buy train ticket \\
\hline Actor & User \\
\hline Requirements & 1) Internet access available \\
& 2) Departure and destination \\
& location should be known by \\
& the user \\
& 3) Valid smartcard number \\
\hline Inputs & 1)Selection of departure and \\
& destination information \\
& 2) Number of persons \\
& child/adult \\
\hline
\end{tabular}




\begin{tabular}{|l|l|}
\hline & $\begin{array}{l}\text { 3) Class of travel } \\
\text { 4) Journey type } \\
\text { (single/return) }\end{array}$ \\
\hline Outputs & $\begin{array}{l}\text { 1) Confirmation message } \\
\text { from } \\
\text { the service } \\
\text { 2) Error message in case of } \\
\text { insufficient balance }\end{array}$ \\
\hline Story & $\begin{array}{l}\text { 1) User views the travel map } \\
\text { after starting the application } \\
\text { 2) User taps on the particular } \\
\text { zone for its zoomed view } \\
\text { 3)User taps and thus selects } \\
\text { the source and destination of } \\
\text { travel } \\
\text { 4) User selects other desired } \\
\text { Information from the } \\
\text { interface } \\
\text { 5) User chooses whether he } \\
\text { wishes for individual tickets } \\
\text { or group ticket if number of } \\
\text { ticket s is greater than one. } \\
\text { 6)User sends request } \\
\text { 7) User receives confirmation } \\
\text { message }\end{array}$ \\
\hline
\end{tabular}

2.1.1.3 Design for Usability : Since usability is the focus of user-centered application, following points are in the spotlight for the touch enabled application:

1) Login with smartcard number

Each user should be able to uniquely login into the system with a smartcard identification number and a password to improve security.

2) Provide option to select Source, Destination and Path

User must be given the choice to choose his/her source, destination and the path through which the ticket is valid.

\section{3) Select destination on map}

User must be able to choose destination by the user interface of Map design, enabling him to click on the location name directly [4].

\section{4) Enlarged screen map}

Maps must contain the feature to zoom in order to view the locations easily [4].

5) Single / return journey

Users may want to purchase tickets in one direction or a return ticket, which includes the travel back to source.

6) Search option for station name

User must be given a choice to search for the location name without having to locate the button on the map.

7) Multilingual

The application must be usable in varied languages.

8) Show map route and integrate with GPS system
User must be given the option to see the exact route from source to destination and user's current position on the map at all times.

The activity flow of the system is shown in Figure 1.

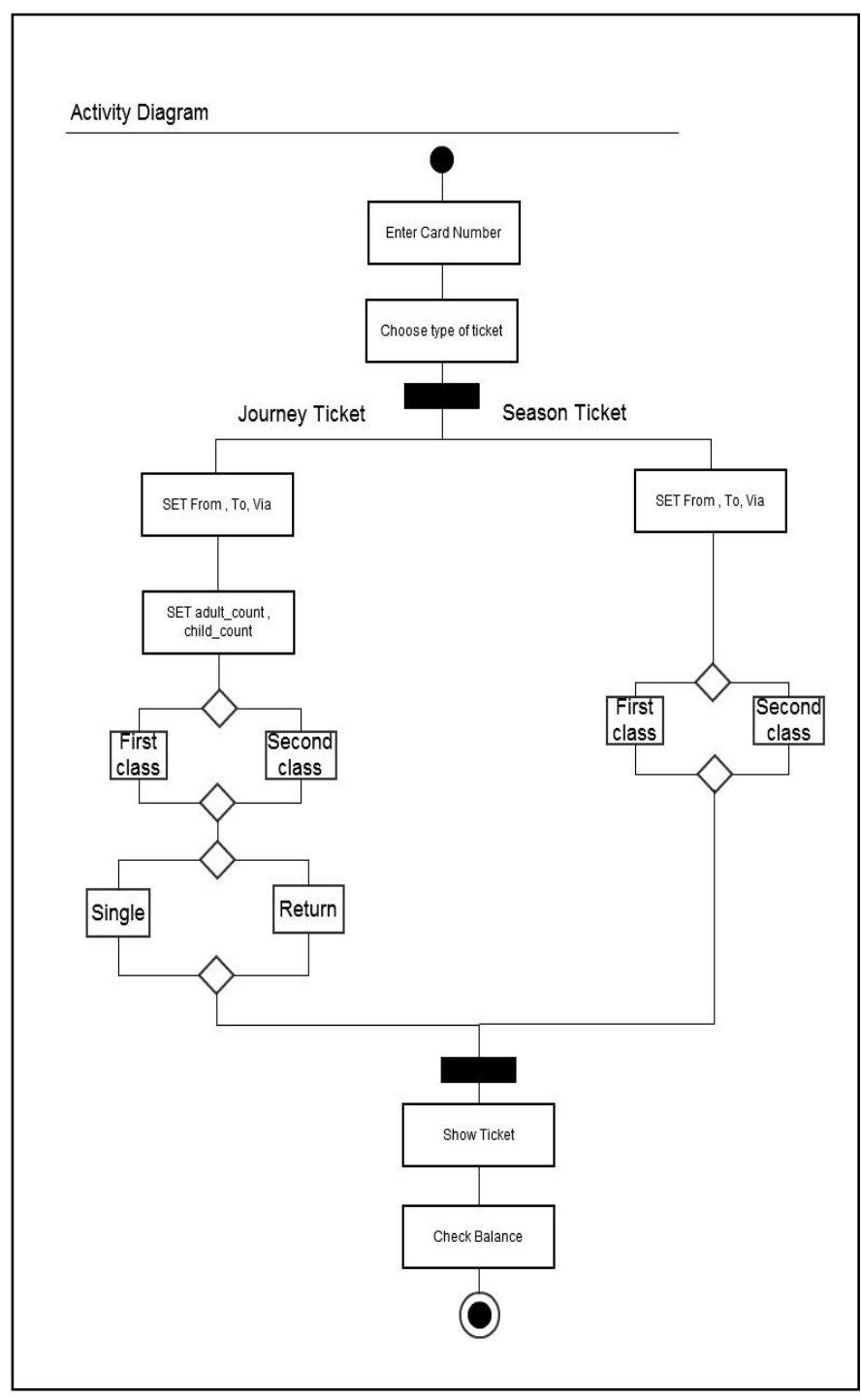

Figure 1. Activity Diagram

2.1.1.4 Construct and Deploy: With the design spotlights in mind, the application is constructed and deployed with the following functionalities :

1) Lucid and intuitive travel map as the first interface.

2) Tap to zoom facility for each zones on the map

3) Tap on source in the map to select source

4) Tap on destination in the map to select destination

5) Appearance of number keypad on tap of number of Adult/Child ticket panel

6) Tap enabled selection of First / Second Class travel

7) Tap enabled selection of Single/Return journey of travel

8) Quick displays of the previous balance fare cost and balance after deduction of fare cost

9) Facility to tap and select group/individual ticket printing

Thus the continuous focus was on users and usability. Usability testing and monitoring was also done in this phase. 
2.1.1.5 Evaluate: Evaluation was started in an early stage and was done continuously. The usability and business effects were continuously tested.

2.1.1.6 Feedback: In the feedback phase plan for the next iteration was done. Suggestions for changes were taken from a set of targeted users as well as the authorities. Based on the outcome, the project was planned.

\section{IMPLEMENTATION}

In the real world implementation it is necessary that the application must be kept up-to-date with the server Database to keep track of any change in location names or prices. An external map application should be used for finding the final destination. Even after the user reaches the destination, the application should allow the user to travel to his final destination using a preferred map application like Google Maps. The ticket received by the user must contain either a barcode uniquely identifying the travel details for the particular user, or a unique identification number that can be easily verified.

The application has been implemented in the Android operating system. Currently, it has been implemented for one of the divisions of the Indian Railways. The features implemented in the said application are:

1) Login with the unique smart card number

2) Map provided to select source and destination

3) Facility to buy ticket for one way and return journey for any number of passengers

4) Search option for finding the required station.

Also, all the selections are on tap and the application on whole is reviewed to be very instinctive. The application has been tested on the Samsung Galaxy 10.1 Tablet. The snapshots are as shown in Figure 2 and Figure 3.

\section{ADDITIONAL ADVANTAGES}

This application is directed towards the public transport users, mainly the bus and railway service users. Apart from the basic aim of usability and functionality, additional advantages of this application are:

\section{A. Faster money Transaction}

1) Following the user interface guidelines of mobile development to increase the ease of use for the general public, it is possible to make the usage of the application completely user-friendly, this making the process of ticketing faster.

2) Also, fast algorithms can reduce the response time for the application to finish the online transaction.

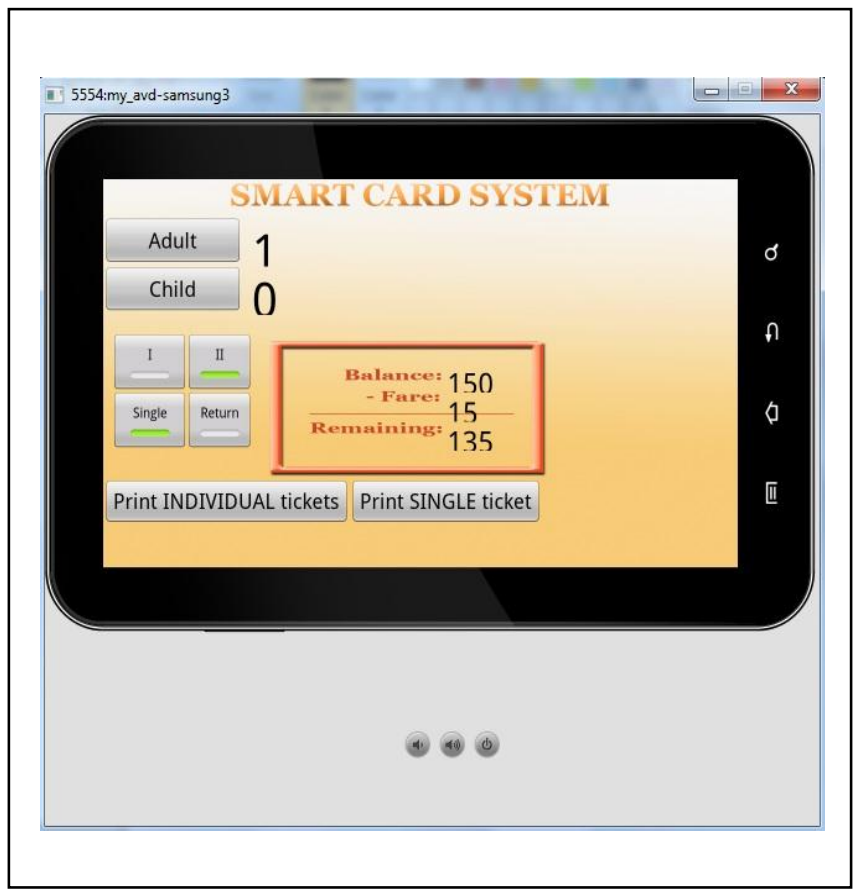

Figure 2. Screen for selecting ticket details

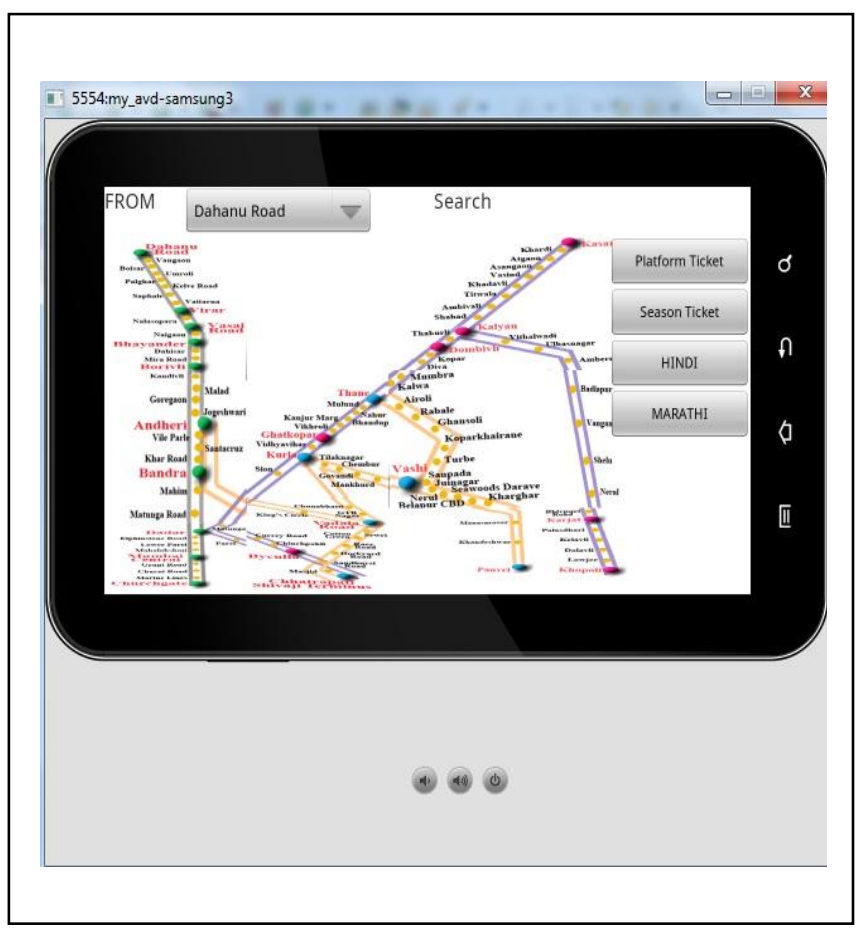

Figure 3. The main screen showing the map 


\section{B. Crowd management}

1) Indian western railways have experienced a huge amount of tickets sold via smartcard in short amount of time. According to a survey held in May, 2011, 38 million tickets have been issued on Smart Cards while 5.8 lakh Smart Cards have been sold since October, 2007[5]. Thus integrating the smart card functionality into the application ensures crowd management.

\section{Validation}

1) Barcodes can be sent as a part of confirmation message to the ticket holders which will be displayed as the ticket on the mobile screen.

2) A barcode scanner typically can record data five to seven times as fast as a skilled typist thus ensuring faster data entry.

3) Keyboard data entry creates an average of one error in 300 keystrokes. Barcode data entry has an error rate of about 1 in 3 million thus ensuring better accuracy.

\section{Eco-friendliness}

Mobile technologies and services can help reduce carbon emission and improve energy efficiency: by the replacement of material goods by non materials substitutes (e-ticketing, ebooks) [6].

Table 2.Comparison with the existing ATVM system

\begin{tabular}{|l|c|c|}
\hline Parameter & $\begin{array}{c}\text { Existing ATVM } \\
\text { Machines }\end{array}$ & $\begin{array}{c}\text { Proposed Smartcard } \\
\text { based Android } \\
\text { Application }\end{array}$ \\
\hline $\begin{array}{l}\text { Provision of } \\
\text { half ticket }\end{array}$ & $\mathrm{x}$ & $\checkmark$ \\
\hline $\begin{array}{l}\text { Need of } \\
\text { separate } \\
\text { first /second } \\
\text { class } \\
\text { validators }\end{array}$ & $\checkmark$ & $\mathrm{x}$ \\
\hline $\begin{array}{l}\text { Ticketing } \\
\text { on-the-go }\end{array}$ & $\mathrm{x}$ & \\
\hline Eco-friendly & $\mathrm{x}$ & \\
\hline $\begin{array}{l}\text { Tap to zoom } \\
\text { interface }\end{array}$ & $\mathrm{x}$ & $\checkmark$ \\
\hline
\end{tabular}

\section{CONCLUSIONS AND FUTURE WORK}

For future, the application can be implemented for all the different mobile operating systems like Android, iOS, Blackberry OS, Mango, etc. While doing so, challenges in visual based HCI will have to be tackled. This is because of the variance in the different operating systems in the area of gesture recognition. The application could be generalized for all transports i.e. one application for all transport systems like bus, railways, road ways (toll plazas) and airlines. There will be a need for adaptivity and personalization, for allowing the use of different input strategies, with responses tailored according to user preferences. This application can also be extended to allow users suffering from certain disabilities. Features like audio and voice recognition facilities can be added [8]. In HCI, Automatic Speech Recognition (ASR) technologies can be used to facilitate this purpose.

The mobile application for public transport ticketing has a huge set of features and can be combined with the smartcard system to efficiently carry out transactions using the advantages of both the systems. This technology will significantly impact the way in which people perceive public transport ticketing options available to them. This opens the door to a myriad of opportunities to enhance the citizen's ticketing experience in terms of usability, functionality and expediency.

\section{REFERENCES}

[1] Dr.Alex Roney Mathew, Mr. Aayad Al Hajj, Mr. Ahmed Al Abri , "Human-Computer Interaction (HCI): An Overview",2011 IEEE International Conference on Computer Science and Automation Engineering (CSAE), pp. 99-100.

[2] Gartner, "Market Share: Mobile CommunicationDevices by Region and Country, 3Q11,”.Viewed Feb. 2012; http://www.gartner.com/resId=1847315.

[3] Winograd, T. "The Design of Interaction," In Beyond Calculation: The Next Fifty Years of Computers, Denning, P.J. \& Metcalfe, R.M. (Eds.) Copernicus, 1997.

[4] Tom Rodden, Keith Chervest, Nigel Davies, Alan Dix, "Exploiting Context in HCI Design or Mobile Systems", Workshop on Human Computer Interaction with Mobile Devices, Pensyllvania State University.

[5] Sharat Chandrayan," Sale Of Atvm Smart Cards Receives Major Boost On WR," press release,22 Jun. 2011;

http://www.wr.indianrailways.gov.in/view_detail.jsp?lan $\mathrm{g}=0 \& \mathrm{id}=0,4,268 \& \mathrm{dcd}=351 \& \mathrm{did}=13087486985628 \mathrm{AD} 83$ 09F37A4A5595E3625AB2F628BB0.web103 .

[6] Knud Erik Skouby, Iwona Windekilde, "Green Mobile Exploring The Environmental Impact Ofstructural Change In Knowledge-Based Economy,"13 Jun.2010 ; GIN2010,Seoul, South Korea.

[7] Informa healthcare, "Speech Recognition technology for individuals with disabilities, Augmentative and Alternative Communication,"Viewed Aug. 2012; "http://informahealthcare.com/doi/abs/10.1080/07434619 212331276333 t”.

[8] Kuo-Ying Huang, "Challenges in Human-Computer Interaction Design for Mobile Devices", Proceedings of the World Congress on Engineering and Computer Science 2009 Vol I WCECS 2009, San Francisco, USA. 